\title{
Searching for Dark Photon Dark Matter with Gravitational-Wave Detectors
}

\author{
Aaron Pierce, ${ }^{1}$ Keith Riles, ${ }^{2}$ and Yue Zhao ${ }^{1,3,4}$ \\ ${ }^{1}$ Leinweber Center for Theoretical Physics, Department of Physics, University of Michigan, Ann Arbor, Michigan 48109, USA \\ ${ }^{2}$ Department of Physics, University of Michigan, Ann Arbor, Michigan 48109, USA \\ ${ }^{3}$ Department of Physics and Astronomy, University of Utah, Salt Lake City, Utah 84112, USA \\ ${ }^{4}$ Tsung-Dao Lee Institute, and Department of Physics and Astronomy, Shanghai Jiao Tong University, Shanghai 200240, China
}

(Received 7 February 2018; revised manuscript received 2 May 2018; published 8 August 2018)

\begin{abstract}
If dark matter stems from the background of a very light gauge boson, this gauge boson could exert forces on test masses in gravitational wave detectors, resulting in displacements with a characteristic frequency set by the gauge boson mass. We outline a novel search strategy for such dark matter, assuming the dark photon is the gauge boson of $U(1)_{B}$ or $U(1)_{B-L}$. We show that both ground-based and future space-based gravitational wave detectors have the capability to make a $5 \sigma$ discovery in unexplored parameter regimes.
\end{abstract}

DOI: 10.1103/PhysRevLett.121.061102

Introduction.-Dark matter (DM) makes up the dominant form of matter in the Universe, but the properties of the particles that compose it remain unknown. If the DM particle is a boson, it can be extremely light, with masses bounded by limits from dwarf galaxy morphology, $m \gtrsim 10^{-22} \mathrm{eV}$, see, e.g., [1]. (We use natural units $c=\hbar=\sqrt{4 \pi \varepsilon_{0}}=1$ but provide in the Appendix a translation of a critical expression to SI units.) A good candidate for ultralight DM is the dark photon (DP), the gauge boson of a simple $U(1)$ extension of the Standard Model. The DP can be naturally light due to its gauge symmetry. The relic abundance must be produced nonthermally; i.e., DPDM remains decoupled from the thermal bath, and so it effectively cools to be nonrelativistic before matter-radiation equality and acts as cold dark matter. There are many possible mechanisms, such as a misalignment mechanism associated with the inflationary epoch, as first discussed in [2], with additional discussion and resolution of subtleties in $[3,4]$. Other nonthermal production mechanisms are possible [5].

When the dark matter is very light, its local occupation number is much larger than one. It can then be treated as a coherently oscillating background field with oscillation frequency determined by its mass $[3,6]$. DPDM therefore imparts external oscillating forces acting on objects carrying nonzero dark charge. While the identity of the DPDM is model dependent, we consider gauged baryon number, $U(1)_{B}$, and baryon number minus lepton number, $U(1)_{B-L}$, as benchmarks in later discussions.

Published by the American Physical Society under the terms of the Creative Commons Attribution 4.0 International license. Further distribution of this work must maintain attribution to the author(s) and the published article's title, journal citation, and DOI. Funded by SCOAP .
The $U(1)_{B}$ is anomalous with SM particle content. Introducing new particles charged under both $U(1)_{B}$ and SM gauge group may cause phenomenological problems $[7,8]$. However, if one applies Green-Schwartz mechanism or mixes the new chiral fermions with particles with large vector mass after electroweak symmetry breaking (like in partial compositeness models [9]), phenomenology constraints can be largely relaxed. The test objects in gravitational-waves (GW) experiments are not exactly charge neutral. This may open the possibility of studying kinetically mixed DPDM [10] .

The strongest constraints on the coupling of light gauge bosons to the Standard Model (SM) come from equivalence principle tests, including those from the Eöt-Wash group $[11,12]$ and Lunar Laser Ranging (LLR) experiments [13-15]. In such experiments, Earth provides a large dark charge, sourcing a dark photon field [16]. Using the EötWash experiment to look for DPDM has been suggested in [17]. See also [18]. Another potential powerful constraint comes from consideration of black hole superradiance, initially proposed in [19] to probe spin-0 particles, such as the axion. It was generalized in [20-23] for a light vector boson. The absence of related signals could rule out some of the regions of interest discussed here. Importantly, however, the effective superradiance requires the absence of nongravitational interactions to a very precise degree [20,24]. Self-interactions can be easily introduced in a dark sector for a massive gauge boson. Indeed, depending on the DPDM production mechanism, such interactions may be expected. We do not discuss these bounds further.

Recent detections of GW by the LIGO and Virgo detectors, see e.g., [25-27], have opened the era of GW astronomy. These interferometers currently measure strain amplitudes of transient GW signals at better than $10^{-21}$, with improvements of a factor of $\sim 3$ expected in the next several years to reach design sensitivities [28]. These strain 
measurements hinge on subattometer sensitivities to the relative displacement of mirrors located 3-4 km apart. As we will discuss, relative displacements of the test masses (interferometer mirrors) may be generated not only by the passage of GW, but also by a DPDM background. A somewhat related idea of using GW detectors to search for clumps of dark matter via the induced displacements has been discussed in $[29,30]$. For longer-lived signals, integration over long observation times can yield strain sensitivities orders of magnitude lower than is possible for transient signals. The DM galactic velocity dispersion is $v_{0} \sim \mathcal{O}\left(10^{-3}\right)$; thus the coherence time is $\sim \mathcal{O}\left(10^{6}\right)$ oscillation periods $\left(10^{6} / f\right)$.

In this Letter, we propose a novel search which can be carried out by GW detectors, presently with the Laser Interferometer Gravitational-Wave Observatory (LIGO) and Virgo and in the future with the Laser Interferometer Space Antenna (LISA). Both ground-based and space-based experiments have the potential to probe an unexplored parameter space of DPDM.

Dark photon dark matter induced relative displacement.-Owing to its light mass, DPDM will be coherent over long length scales. Its spatial coherence length can be estimated as $\ell_{\text {coherence }}=2 \pi /\left(m_{A} v_{0}\right)$, where $m_{A}$ is the mass of the dark photon, and $v_{0}$ corresponds to a typical dark matter virial velocity in the halo, $v_{0} \sim 10^{-3}$.

For frequencies corresponding to near the best sensitivity of LIGO, $m_{A}=2 \pi f=2 \pi(100 \mathrm{~Hz})=4 \times 10^{-13} \mathrm{eV}$, and $v_{0}=10^{-3}$, we have $\ell_{\text {coherence }} \simeq 3 \times 10^{6} \mathrm{~km}$.

The local amplitude $A_{\mu, 0}$ of the dark gauge field $A_{\mu}$ can be found by equating its energy density, $\frac{1}{2} m_{A}^{2} A_{\mu, 0} A_{0}^{\mu}$, to that of the local dark matter, for which we take a fiducial value of $\rho_{\mathrm{DM}}=0.4 \mathrm{GeV} / \mathrm{cm}^{3}$. Within a coherence length, $A_{\mu}(t, \vec{x}) \simeq$ $A_{\mu, 0} \sin \left(m_{A} t-\vec{k} \cdot \vec{x}\right)$. This oscillating dark photon field will act as an external force on the test objects of GW detectors, and the resulting displacements may be detected by such experiments.

Since the DPDM is nonrelativistic, the electric components associated with the time derivative of the field are much larger than the magnetic components. The acceleration acting on a test mass located at $x_{i}$ is

$$
\begin{aligned}
\vec{a}_{i}\left(t, \vec{x}_{i}\right) & =\frac{\vec{F}_{i}\left(t, \vec{x}_{i}\right)}{M_{i}} \simeq \epsilon e \frac{q_{D, i}}{M_{i}} \partial_{t} \vec{A}\left(t, \vec{x}_{i}\right) \\
& =\epsilon e \frac{q_{D, i}}{M_{i}} m_{A} \vec{A}_{0} \cos \left(m_{A} t-\vec{k} \cdot \vec{x}_{i}\right) .
\end{aligned}
$$

We normalize the coupling of the dark photon in terms of the electromagnetic (EM) coupling constant $e$. The ratio of the dark photon coupling strength to the EM coupling strength is given by $\epsilon$, which is not constrained theoretically. $M_{i}$ and $q_{D, i}$ are the total mass and dark charge of the $i$ th test object. If the dark photon is a gauge field associated with baryon number, $U(1)_{B}, q_{D}$ is the total baryon number; for $U(1)_{B-L}, q_{D}$ counts the neutrons in the material.
A GW detector is sensitive to the differential relative displacement between pairs of test objects along different axes. This displacement will be induced by slightly differing forces on the test masses, a difference determined by the relative phase of the dark photon field at the positions of the test objects.

For dark photon masses we consider, this phase difference is small and results in a suppression by the small virial velocity $v_{0}$. The arm lengths are $4 \mathrm{~km}$ and $2.5 \times 10^{6} \mathrm{~km}$ for LIGO and LISA. For $v_{0}=O\left(10^{-3}\right)$, as long as the dark photon oscillation frequency is smaller than $\mathcal{O}\left(10^{8}\right) \mathrm{Hz}$ $\left[\mathcal{O}\left(10^{2}\right) \mathrm{Hz}\right]$ for LIGO (LISA), the arm length is always much smaller than the wavelength of the dark photon background. In contrast, the best sensitivities of these experiments are at $\mathcal{O}\left(10^{2}\right) \mathrm{Hz} \mathcal{O}\left(10^{-2}\right) \mathrm{Hz}$. Thus $\left|\vec{k} \cdot\left(\vec{x}_{1}-\vec{x}_{2}\right)\right| \ll 1$ is a good approximation in the frequency regimes with the best sensitivity in both experiments.

With this approximation, and noting the test object pairs are composed of the same elements, i.e., they have the same $\left(q_{D, i} / M_{i}\right)$, the amplitude of the induced differential strain in one Michelson interferometer (relative displacement $\Delta L$ divided by arm length $L$ ) can be calculated. Following the details shown in the Appendix, we have

$$
R \equiv \frac{\Delta L}{L} \simeq C \frac{q_{D}}{M} \epsilon e\left|\vec{A}_{0}\right| v_{0} .
$$

Here $C$ is the geometric factor found by averaging over the direction of DM propagation and the dark photon polarization, accounting for the orientation of the GW detector arms. This is generically $\mathcal{O}(1)$. In the Appendix, we explicitly show $C_{\mathrm{LIGO}}=(\sqrt{2} / 3)$ and $C_{\mathrm{LISA}}=(1 / \sqrt{6})$. For a $U(1)_{B}$ and $U(1)_{B-L}$ gauge boson acting on a mirror composed of Silicon, $\left(q_{D} / M\right) \simeq(1 / \mathrm{GeV})$ and $(1 / 2 \mathrm{GeV})$, respectively. To arrive at Eq. (2), we use the instantaneous acceleration of Eq. (1), and compute the displacement as a function of time.

Experimental sensitivity to a near-monochromatic stochastic $G W$ background.-While the oscillation frequency of the DPDM field is determined by the dark photon mass, the virial velocity broadens the oscillation frequency, i.e., $\Delta f / f \sim v_{0}^{2}$. Since $v_{0}$ is $\mathcal{O}\left(10^{-3}\right)$, the signal is nearly monochromatic.

In this section, we begin by examining the experimental sensitivity of a GW detector to a near-monochromatic stochastic gravitational wave. We will then rephrase this monochromatic GW sensitivity in terms of a limit on the dark photon. We emphasize that this is a calculational tool; no gravitational waves are present.

A sinusoidal, linearly polarized gravitational plane wave with frequency $f$ and strain $h(\vec{r}, t)$, has energy density [31]

$$
\rho_{\mathrm{GW}}(f)=\frac{\left\langle\dot{h}^{2}\right\rangle}{16 \pi G}=(2 \pi f)^{2} \frac{\left\langle h^{2}\right\rangle}{16 \pi G} .
$$

Here the average is over time at a given point. For a plane wave with amplitude $h_{0},\left\langle h^{2}\right\rangle=\frac{1}{2} h_{0}^{2}$. The one-sided power spectrum of GW strain for such a near-monochromatic 
GW can be written in the customary form in terms of the fraction of the critical density attributable to gravitational waves [32]:

$$
S_{\mathrm{GW}}(f)=\frac{3 H_{0}^{2}}{2 \pi^{2}} f^{-3} \Omega_{\mathrm{GW}}(f),
$$

with

$$
\Omega_{\mathrm{GW}}(f) \equiv \frac{f}{\rho_{c}} \frac{d \rho_{\mathrm{GW}}}{d f}=\frac{f}{\rho_{c}} \frac{\Delta \rho_{\mathrm{GW}}(f)}{\Delta f},
$$

where the critical density $\rho_{c}$ is related to the Hubble constant $H_{0}$ as $\rho_{c}=\left(3 H_{0}^{2} / 8 \pi G\right)$, and we have

$$
S_{\mathrm{GW}}(f)=\frac{h_{0}^{2}}{2 \Delta f} .
$$

In Eq. (5), we specialized to the frequency window $\Delta f$ where the signal (stochastic GW or DPDM) would lie.

The detection of a stochastic cosmological background with a single detector is difficult, because it may be indistinguishable from other unknown sources of noise. Using cross-correlation between comparable, independent interferometers, however, permits a dramatically better sensitivity via integration of the correlation over time. To calculate the achievable signal-to-noise ratio (SNR) for a near-monochromatic GW signal, we follow the analogous SNR calculation for LIGO broadband stochastic searches based on cross-correlation of GW strain signals between different interferometers [33].

The expectation value and variance of the standard stochastic GW detection statistic can be written as

$$
\begin{aligned}
S & =\frac{T}{2} \int d f \gamma(|f|) S_{\mathrm{GW}}(|f|) \tilde{Q}(f), \\
N^{2} & =\frac{T}{4} \int d f P_{1}(|f|)|\tilde{Q}(f)|^{2} P_{2}(|f|) .
\end{aligned}
$$

The SNR is $S / N . T$ is the total observing time of the GW experiment, and we take $T=2$ years. $\gamma(|f|)$ is the overlap reduction function between two GW detectors [34], e.g., the LIGO Hanford and Livingston interferometers. $\tilde{Q}(f)$ is the Fourier transform of the optimal filter function, and $P_{1,2}(f)$ are the one-sided strain noise power spectra of the two detectors.

For a given signal $S_{\mathrm{GW}}(|f|), \tilde{Q}(f)$ should take the following form in order to maximize SNR, see [35] for a derivation:

$$
\tilde{Q}(f)=\mathcal{N} \frac{\gamma(|f|) S_{\mathrm{GW}}(|f|)}{P_{1}(|f|) P_{2}(|f|)} .
$$

$\mathcal{N}$ is the normalization factor, which will be dropped when calculating SNR. For a near-monochromatic GW with width $\Delta f$, we then find

$$
\mathrm{SNR}=\frac{\gamma(|f|) h_{0}^{2} \sqrt{T}}{2 \sqrt{P_{1}(f) P_{2}(f) \Delta f}}
$$

Comparison of a DPDM search with a stochastic GW search.-Interferometer response to a dark photon dark matter field is similar to that to a stochastic gravitational wave background, hence the similarities in analysis methods described in the preceding section. There are some important differences to keep in mind, however. Most important are (1) the inherently long coherence length of the DPDM signal, which ensures a strong simultaneous correlation in the interferometer responses, and (2) the long coherence time $\left(\sim 10^{6} / f\right.$, or about $10^{4}$ sec for a $100-\mathrm{Hz}$ signal), which restricts the bandwidth of the signal, permitting a high signal-to-noise ratio.

For a stochastic GW signal, the overlap function is $\mathcal{O}(1)$ at long wavelength and falls off for shorter wavelengths, set by the separation between the two detectors. $\gamma(|f|)$ falls off rapidly above $\sim 10 \mathrm{~Hz}$ for Hanford and Livingston. For our signal, the coherence length is enhanced by $1 / v$, so the falloff in $\gamma(|f|)$ is unimportant below $\sim 10^{4} \mathrm{~Hz}$, well above the best sensitivity. This implies $|\gamma|$ near unity for the Hanford and Livingston interferometers, which are, by design, nearly aligned with each other, albeit with a rotation by $90^{\circ}$ that introduces a relative sign flip in $\Delta L$ and with a misalignment of the normal vectors to the planes of the interferometers by $27^{\circ}$. As a result, the normalized overlap reduction function, averaged over all directions of the wave vector $\vec{k}$ and field polarization $\vec{A}$, is -0.9 for the Hanford and Livingston interferometers. The overlap reduction functions for the three pairs of LISA Michelson interferometers are also $\mathcal{O}(1)$, but instrumental correlations require construction of synthetic noise-orthogonal interferometers for cross-correlation signal extraction. We follow the treatment of [36] in using the " $\langle A E\rangle$ " correlation for which we estimate a normalized overlap reduction function of -0.29 .

For the DPDM signal, the dark matter velocity distribution would be well modeled as a Maxwell-Boltzmann distribution with a cutoff at the escape velocity:

$$
f(\vec{v}) \propto e^{-|v|^{2} / v_{0}^{2} \Theta\left(v_{\mathrm{esc}}-|v|\right) .}
$$

We take $v_{0}=230 \mathrm{~km} / \mathrm{s}$, and $v_{\text {esc }}=544 \mathrm{~km} / \mathrm{sec}$, the central value given by the RAVE Collaboration [37]. In frequency space, the signal will be peaked around $\omega=\sqrt{m^{2}+k^{2}} \approx m\left(1+\left(v_{0}^{2} / 2\right)\right)$, with a falloff controlled by the above distribution. In our calculations, we choose $v \in\left\{0.2 v_{0}, 1.8 v_{0}\right\}$ in order to include $90 \%$ of the DM energy density. This implies $\Delta f / f \simeq 0.95 \times 10^{-6}$.

These $\Delta f$ are even smaller than the bandwidths of typical continuous wave sources sought from fast-spinning, nonaxisymmetric neutron stars in the galaxy, for which Doppler modulations from Earth's orbit lead to frequency spreads of $\pm \mathcal{O}\left(10^{-4}\right) f$ over the course of a year [38]. [In the case of our signal, the Doppler effect leads to a small modulated broadening of $\mathcal{O}\left(10^{-7}\right)$.] Previous directed GW searches using stochastic analysis techniques, e.g., from the 
Supernova 1987A remnant or from the Galactic Center, have used coarser $\Delta f$ binning than is necessary in a DPDM search [39]. Those directed GW searches have benefitted from knowing a priori the phase difference between a pair of interferometers with respect to a fixed direction on the sky. In the DPDM search that phase difference is nearly zero because of the long coherence length of the field.

For current GW observatories, this paper focuses on how a correlation between the nominally identical and nearly aligned Hanford and Livingston interferometers can be exploited at Advanced LIGO design sensitivities. The Virgo interferometer operating at design sensitivity would potentially offer improved sensitivity when used in a network cross correlation. The gain will be modest, however, because the intrinsic Virgo sensitivity is expected to be worse than LIGO and the normalized overlap reduction functions with respect to the LIGO interferometers are quite low in magnitude ( -0.02 for Hanford-Virgo and -0.25 for Livingston-Virgo). Virgo could, however, play a useful role in confirming a statistically significant outlier found in LIGO analysis; a loud-enough outlier found in Hanford-Livingston cross-correlation could be visible with lower strength in the Livingston-Virgo correlation. In addition, the Virgo interferometer is different enough in design from the LIGO interferometers that nonGaussian, instrumental spectral lines correlated between Hanford and Livingston, which are extremely difficult to eliminate entirely, given nominally identical electronics, are less likely to occur at the same frequencies in Virgo. A notable example is electrical power mains, which unavoidably contaminate $\mathrm{GW}$ strain data at some level and operate in the U.S. at $60 \mathrm{~Hz}$ and in Europe at $50 \mathrm{~Hz}$. A detailed analysis of how to exploit LIGO and Virgo correlations is beyond the scope of this article. See [40] for a network stochastic analysis combining Initial LIGO and Virgo data.

Results.-For a given choice of SNR, one can estimate the minimal value of "GW amplitude" $h_{0}$ detectable by a GW experiment, setting $\Delta f$ as described above for our DPDM signal with long coherence time. In order to translate the limit on $h_{0}$ to the expected sensitivity on the dark photon coupling strength normalized to EM coupling strength $\epsilon^{2}=\alpha_{D} / \alpha_{\mathrm{EM}}$, we need to compare $h_{0}$ with the relative displacement $R$ in Eq. (2). The passage of a GW plane wave with magnitude $h_{0}$ is equivalent to a relative displacement with $R=h_{0} / 2$.

We consider both exclusion limits, as well as discovery potential. In the absence of a signal, DPDM can be constrained. Following convention, we set $\mathrm{SNR}=2$ to set the limit as a function of frequency. A $5 \sigma$ local significance (i.e., after including a trials factor) is quoted as a benchmark for discovery. Since our signal is almostmonochromatic, i.e., $\Delta f / f \sim 10^{-6}$, this is effectively a bump-hunt in frequency space, and the trials factor is $\mathcal{O}\left(10^{6}\right)$. We therefore take SNR $\approx 7$ for discovery.

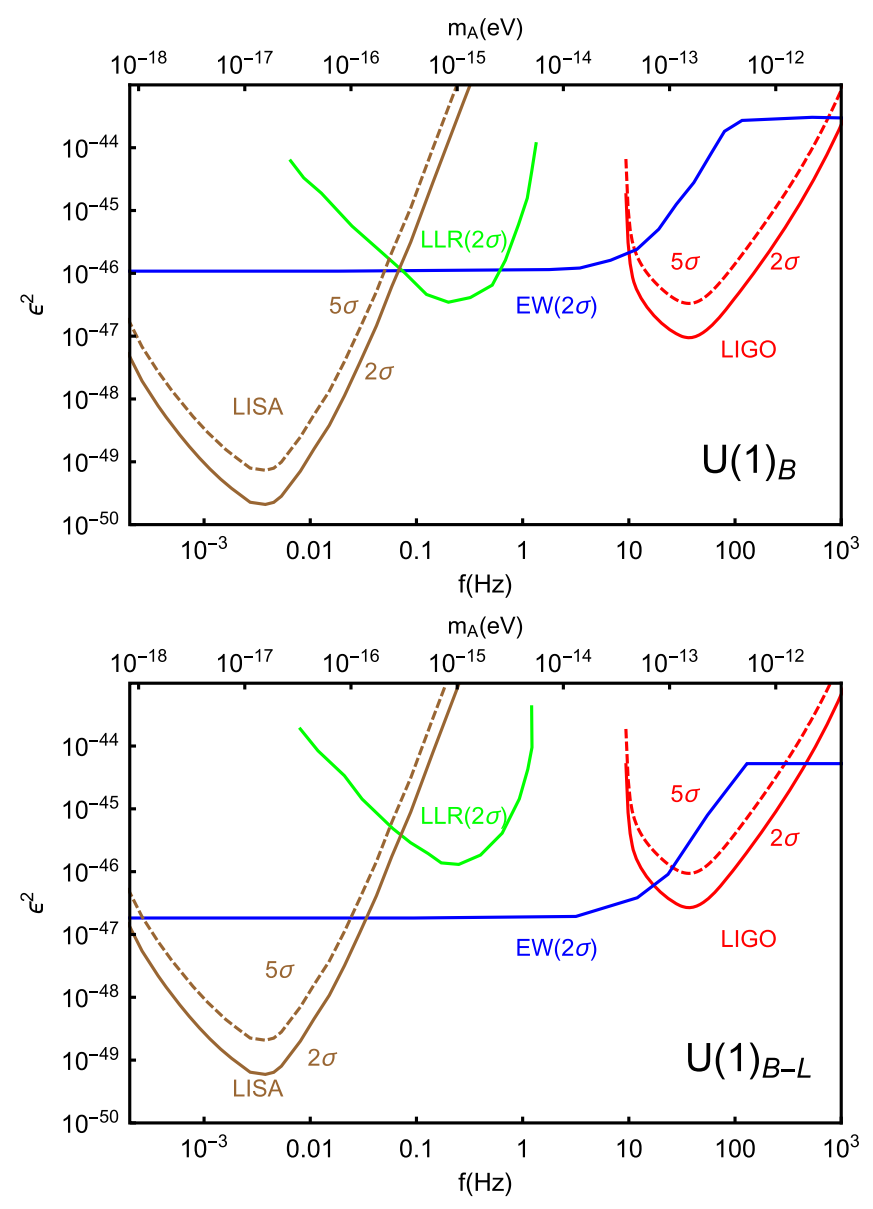

FIG. 1. The $2 \sigma$ exclusion limit and $5 \sigma$ discovery potential obtained from LIGO and LISA after $2 \mathrm{yr}$ of coincident running for $B$ (upper) and $(B-L)$ (lower) dark photon dark matter. Coupling strength is normalized to EM coupling strength, i.e., $\epsilon^{2}=\alpha / \alpha_{\mathrm{EM}}$, which is not constrained theoretically. The blue and green curves are limits from the Eöt-Wash (EW) experiment [11,12] and the Lunar Laser Ranging (LLR) experiment [13-15]. The idealized design LIGO sensitivity curves used here do not include very narrow bands of instrumental line artifacts, such as from $60-\mathrm{Hz}$ power mains contamination and vibration modes of mirror suspension fibers, for which DPDM sensitivity is degraded $[42,43]$.

In Fig. 1 , we show $2 \sigma$ exclusion limits and $5 \sigma$ discovery potentials in the $\epsilon^{2}$-frequency plane, assuming the dark $U(1)$ is the $B$ and $(B-L)$ group, for the LIGO and LISA experiments. We approximate the LIGO and LISA mirrors as being composed of silica. $T$ is set to $2 \mathrm{yr}$ and $|\gamma(|f|)|$ is chosen to be 0.9 and 0.29 for LIGO and LISA, respectively. The one-sided strain noise power spectra for LIGO and LISA are taken from [28,41], with the frequency window set as described below Eq. (10).

Conclusion.-We have shown that GW detectors are potentially sensitive to the presence of a light gauge field acting as the dark matter. Present Earth-based interferometers may place the strongest bounds on $U(1)_{B-L}$ and $U(1)_{B}$ gauge fields near their peak sensitivity of $\mathcal{O}(100) \mathrm{Hz}$ 
$\left(m_{A} \approx 4 \times 10^{-13} \mathrm{eV}\right)$, and in the case of $U(1)_{B}$, these experiments have $5 \sigma$ discovery potential. LISA should make comparable progress in the region of its peak sensitivity, $\mathcal{O}\left(10^{-2}\right) \mathrm{Hz} \quad\left(m_{A} \approx 4 \times 10^{-17} \mathrm{eV}\right)$ [44]. Unlike other bounds on light gauge fields, these limits are sensitive to the usual astrophysical uncertainties on the distribution of the dark matter. Variations in the local dark matter density will directly impact the strength of the bound, as can variation of the velocity dispersion of the DM; see Eq. (2). For a veryhigh-SNR detection of DPDM (allowed for LISA and for a third-generation ground-based detector by current experimental constraints), the signal's spectral line shape would yield the dark matter speed distribution, and the signal strength's time dependence would yield directional information, including self-consistency checks.

The authors would like to thank M. Arvanitaki and Y. Zhong for useful discussions and E. Thrane for valuable comments on the manuscript. A. P. and Y.Z. are supported by the U.S. Department of Energy under Grant No. DESC0007859. K. R. is supported by the U.S. National Science Foundation under Grant No. NSF-PHY1505932. Y.Z. also thanks to the Department of Physics \& Astronomy, University of Utah and the Office of Science and Technology, Shanghai Municipal Government (No. 16DZ2260200).

\section{APPENDIX: DETAILS ON THE GEOMETRIC FACTORS}

Here, we compute the geometric factor $C$, see Eq. (2), that characterizes the relative orientations of interferometer arms and the incident dark matter. Since the dark photon dark matter is nonrelativistic, there is no correlation between the direction of propagation and the polarization of the gauge field.

For concreteness we will first focus on LIGO where two arms are orthogonal to each other, and we choose them to be the $x$ and $y$ axes. The GW detector effectively measures the relative change of two arm lengths, i.e., $\left(\Delta L_{x}-\Delta L_{y}\right)$. This can be calculated from Eq. (1) as

$$
\begin{aligned}
& \left(\Delta L_{x}-\Delta L_{y}\right) \\
& =\int d t \int d t\left\{a_{x}\left[\cos \left(m_{A} t-\vec{k} \cdot \vec{x}_{1}\right)-\cos \left(m_{A} t-\vec{k} \cdot \vec{x}_{2}\right)\right]\right. \\
& \left.\quad-a_{y}\left[\cos \left(m_{A} t-\vec{k} \cdot \vec{y}_{1}\right)-\cos \left(m_{A} t-\vec{k} \cdot \vec{y}_{2}\right)\right]\right\}
\end{aligned}
$$

where $a_{x}$ and $a_{y}$ are the accelerations along the $x$ and $y$ axes. $\vec{x}_{1,2}$ and $\vec{y}_{1,2}$ are the position vectors of test masses and $L$ is the arm length at LIGO: $\left|\vec{x}_{1}-\vec{x}_{2}\right|=\left|\vec{y}_{1}-\vec{y}_{2}\right|=L$. Defining the angle between the wave vector $\vec{k}$ and the normal to the LIGO plane as $\alpha$, and the angle between the projected 2D wave vector and the $x$ axis as $\theta$, the amplitude of the oscillating differential displacement of two arms is

$$
\begin{aligned}
\Delta L & \equiv\left|\Delta L_{x}-\Delta L_{y}\right|_{\max } \\
& \simeq\left|a_{x} \cos \theta-a_{y} \sin \theta\right|\left(\frac{|k| L \sin \alpha}{m_{A}^{2}}\right) .
\end{aligned}
$$

We need to perform the average over all possible directions of $\vec{k}$ and $\vec{a}$ (the latter is related to the polarization vector of $A)$. We calculate $\sqrt{\left\langle\Delta L^{2}\right\rangle_{\text {LIGO }}}$, where the \langle\rangle corresponds to this averaging procedure. This gives

$$
\sqrt{\left\langle\Delta L^{2}\right\rangle_{\text {LIGO }}}=\frac{\sqrt{2}}{3} \frac{|a||k| L}{m_{A}^{2}}
$$

where $a$ is the magnitude of acceleration given in Eq. (1). The geometric factor of Eq. (2) is thus $C_{\mathrm{LIGO}}=(\sqrt{2} / 3)$.

A similar calculation can be done for LISA where the opening angles among the three arm pairs are $\pi / 3$, giving $C_{\mathrm{LISA}}=(1 / \sqrt{6})$ for each single interferometer.

From Eq. (1), and using $\rho_{\mathrm{DM}} \simeq \frac{1}{2} m_{A}^{2} A_{\mu, 0} A^{\mu, 0}$, we can write $\Delta L$ in SI units as

$$
\sqrt{\left\langle\Delta L^{2}\right\rangle}=C \frac{\hbar^{2} \epsilon|k| L}{m_{A}^{2} c^{4}} \frac{e q}{M \sqrt{\varepsilon_{0}}} \sqrt{2 \rho_{\mathrm{DM}}} .
$$

[1] S. R. Chen, H. Y. Schive, and T. Chiueh, Mon. Not. R. Astron. Soc. 468, 1338 (2017).

[2] A. E. Nelson and J. Scholtz, Phys. Rev. D 84, 103501 (2011).

[3] P. Arias, D. Cadamuro, M. Goodsell, J. Jaeckel, J. Redondo, and A. Ringwald, J. Cosmol. Astropart. Phys. 06 (2012) 013.

[4] P. W. Graham, J. Mardon, and S. Rajendran, Phys. Rev. D 93, 103520 (2016).

[5] R. T. Co, L. J. Hall, and K. Harigaya, Phys. Rev. Lett. 120, 211602 (2018)

[6] J. Preskill, M. B. Wise, and F. Wilczek, Phys. Lett. 120B, 127 (1983); 120B, 127 (1983).

[7] J. A. Dror, R. Lasenby, and M. Pospelov, Phys. Rev. Lett. 119, 141803 (2017).

[8] J. A. Dror, R. Lasenby, and M. Pospelov, Phys. Rev. D 96, 075036 (2017).

[9] D. B. Kaplan, Nucl. Phys. B365, 259 (1991).

[10] A. Pierce, K. Riles, and Y. Zhao (to be published).

[11] Y. Su, B. R. Heckel, E. G. Adelberger, J. H. Gundlach, M. Harris, G. L. Smith, and H. E. Swanson, Phys. Rev. D 50, 3614 (1994).

[12] S. Schlamminger, K.-Y. Choi, T. A. Wagner, J. H. Gundlach, and E. G. Adelberger, Phys. Rev. Lett. 100, 041101 (2008).

[13] J. G. Williams, S. G. Turyshev, and D. H. Boggs, Phys. Rev. Lett. 93, 261101 (2004).

[14] C. Talmadge, J. P. Berthias, R. W. Hellings, and E. M. Standish, Phys. Rev. Lett. 61, 1159 (1988).

[15] S. G. Turyshev and J. G. Williams, Int. J. Mod. Phys. D 16, 2165 (2007). 
[16] These constraints could in principle be evaded in nonminimal scenarios wherein Earth captured background particles charged under the dark gauge group, thereby screening the charge.

[17] P. W. Graham, D. E. Kaplan, J. Mardon, S. Rajendran, and W. A. Terrano, Phys. Rev. D 93, 075029 (2016).

[18] This paper briefly discussed the possibility for LIGO searching for DPDM; however, many important details such as spin/direction average and correlations among different detectors are left to be studied.

[19] A. Arvanitaki, S. Dimopoulos, S. Dubovsky, N. Kaloper, and J. March-Russell, Phys. Rev. D 81, 123530 (2010).

[20] M. Baryakhtar, R. Lasenby, and M. Teo, Phys. Rev. D 96, 035019 (2017).

[21] W. E. East and F. Pretorius, Phys. Rev. Lett. 119, 041101 (2017).

[22] W. E. East, Phys. Rev. D 96, 024004 (2017).

[23] V. Cardoso, . J. C. Dias, G. S. Hartnett, M. Middleton, P. Pani, and J. E. Santos, J. Cosmol. Astropart. Phys. 03 (2018) 043.

[24] A. Arvanitaki, M. Baryakhtar, and X. Huang, Phys. Rev. D 91, 084011 (2015).

[25] B. P. Abbott et al. (LIGO Scientific and Virgo Collaborations), Phys. Rev. Lett. 116, 061102 (2016).

[26] B. P. Abbott et al. (LIGO Scientific and Virgo Collaborations), Phys. Rev. Lett. 119, 141101 (2017).

[27] B. P. Abbott et al. (LIGO Scientific and Virgo Collaborations), Phys. Rev. Lett. 119, 161101 (2017).

[28] B. P. Abbott et al. (LIGO Scientific and VIRGO Collaborations), Living Rev. Relativity 19, 1 (2016).
[29] A. W. Adams and J. S. Bloom, arXiv:astro-ph/0405266.

[30] E. D. Hall, T. C. V. V. Frolov, T. Callister, H. Muller, M. Pospelov, and R. X. Adhikari, arXiv:1605.01103.

[31] S. L. Shapiro and S A. Teukolsky, Black Holes, White Dwarfs, and Neutron Stars (John Wiley \& Sons, Inc., New York, 1983).

[32] E. Thrane and J. D. Romano, Phys. Rev. D 88, 124032 (2013).

[33] B. Abbott et al. (LIGO Scientific Collaboration), Phys. Rev. D 69, 122004 (2004).

[34] N. Christensen, Phys. Rev. D 46, 5250 (1992).

[35] B. Allen, arXiv:gr-qc/9604033.

[36] J. D. Romano and N. J. Cornish, Living Rev. Relativity 20, 2 (2017).

[37] M. C. Smith et al., Mon. Not. R. Astron. Soc. 379, 755 (2007).

[38] K. Riles, Mod. Phys. Lett. A 32, 1730035 (2017).

[39] B. P. Abbott et al. (LIGO Scientific and Virgo Collaborations), Phys. Rev. Lett. 118, 121102 (2017).

[40] J. Aasi et al. (LIGO Scientific and VIRGO Collaborations), Phys. Rev. Lett. 113, 231101 (2014).

[41] N. Cornish and T. Robson, J. Phys. Conf. Ser. 840, 012024 (2017).

[42] P. Covas et al., Phys. Rev. D 97, 082002 (2018).

[43] B. P. Abbott et al. (LIGO Scientific and Virgo Collaborations), Phys. Rev. D 97, 102003 (2018).

[44] The weak gravity conjecture requires the existence of a particle with $\epsilon q>m / M_{p l}$. This can be satisfied by introducing a $\mathcal{O}(\mathrm{keV})$ particle with $\epsilon \sim 10^{-25}$. 